\title{
Regulation of Adult Neurogenesis in the Cerebral Cortex Koji Ohira*
}

Laboratory of Nutritional Brain Science, Department of Food Science and Nutrition, Mukogawa Women's University, Nishinomiya, Japan

Article Info

\section{Article Notes}

Received: June 05, 2018

Accepted: August 07, 2018

\section{*Correspondence}

Dr. Koji Ohira, Laboratory of Nutritional Brain Science, Department of Food Science and Nutrition, Mukogawa Women's University, Nishinomiya, Japan; Telephone No: +81 79845 9898; Fax No: +81 79845 9898;

E-mail: kohira@mukogawa-u.ac.jp

C 2018 Ohira K. This article is distributed under the terms of the Creative Commons Attribution 4.0 International License

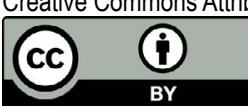

\section{Keywords}

Cerebral cortex

Neurogenesis

Progenitor cell

Stem cell

\section{Abstract}

It is widely accepted that new neurons are generated throughout life, which is called adult neurogenesis, in limited regions of the adult mammalian brain, such as the hippocampal dentate gyrus and subventricular zone. In these regions, neural stem cells (NSCs) and neural progenitor cells (NPCs) have been reported to proliferate and produce postmitotic neurons. Adult neurogenesis in these regions is influenced by various factors. For examples, antidepressant treatments, learning and memory, and environmental enrichment prompt to increase generation and survival of new neurons. Moreover, pathological processes, such as neuroinflammation, stroke or epilepsy, are able to induce proliferation and differentiation of NSCs and NPCs. In contrast, down-regulation of adult neurogenesis is associated with alcohol abuse, high stress level, some drugs, such as cytostatics, COX-2 inhibitors, and opioides. Recently, adult neurogenesis in the cerebral cortex is becoming clear gradually, and cortical NSCs and NPCs are identified in a few mammals. However, it remains largely unknown what factors can regulate adult neurogenesis in the cerebral cortex. This review focuses on the effects of regulating factors on cortical adult neurogenesis, such as brain damages, aging and certain drugs, and we discuss implications of cortical adult neurogenesis for brain diseases and damages.

\section{Introduction}

There are many reports that adult neurogenesis occurs in the subventricular zone (SVZ) and hippocampal dentate gyrus (HDG) of mammalian brains since the $1960 \mathrm{~s}^{1,2}$. Adult neurogenesis in the SVZ and HDG has been reported to be regulated by some regulatory factors, such as stress, environmental enrichment, exercise, certain drugs, aging, and so on. On the other hand, it remains controversial whether adult neurogenesis occurs in other regions of the central nervous system (CNS). In the last decade, cortical adult neurogenesis and its neural stem cells (NSCs), which are self-renewing and can generate neurons, astrocytes and oligodendrocytes, and neural progenitor cells (NPCs), which have a tendency to differentiate into certain types of neurons, have been found in the cerebral cortex of adult mammals ${ }^{3}$. Interestingly, production of new neurons in the cerebral cortex, as well as in the SVZ and HDG, has been found to be strongly increased by brain ischemia ${ }^{3}$. Moreover, certain drugs, such as antidepressants, can accelerate production of neurons from cortical NPCs, and increased neurons from cortical NPCs have the inhibitory effect on neuronal cell death, which is induced by global ischemia ${ }^{4}$. In this review, I summarize recent studies of cortical adult neurogenesis. In particular, I focus on regulating factors of cortical adult neurogenesis. Furthermore, I discuss future research directions for achieving maintenance and treatments for diseases and damages of the cerebral cortex. 


\section{Neurogenesis in the Cerebral Cortex of Adult} Mammals

Historically, the discovery of cortical adult neurogenesis is old, and identifying generation of new cells in the adult cerebral cortex was first reported in $1912^{5}$. After that, using the DNA synthesis marker tritiated thymidine, Altman rediscovered the addition of new neurons in the cerebral cortex of adult rats ${ }^{6}$. Thereafter, using a combination of autoradiography and electron microscopy, Kaplan demonstrated that new cells containing tritiated thymidine are stellate cells that have an axonal hillock, initial segment, and synapses on their dendrites and cell bodies in the cerebral cortex of adult rats ${ }^{7}$. Furthermore, in the last decade, several studies have found NSCs and NPCs capable of generating new neurons in mammals, including mice, rats, and humans.

\section{Distribution of Cortical NSCs and NPCs and Brain Damage-Dependent Cortical Neurogenesis}

Previous reports demonstrate that the percentages of new neurons to total neurons are very small under healthy conditions, and it is in the range of $0.005-0.03 \%$ of all existing neurons in the cerebral corte ${ }^{8-12}$. Interestingly, brain damages can up-regulate the production of new neurons by a factor of $0.06-1 \%$ of total cortical neurons $s^{4,13-16}$. These reports indicate that cortical NSCs and NPCs may produce neurons in a damage or stimulationdependent manner. In addition, emerging evidence has suggested that there seem to be cortical NSCs and NPCs, which can generate new neurons in the cerebral cortex by brain damages, in several subregions of the cerebral cortex, such as the anterior $\mathrm{SVZ}^{8,10,15,17,18}$, white matter ${ }^{19,20}$, gray matter ${ }^{9}$, marginal zone ${ }^{16,20,21}$, perivascular regions ${ }^{22}$, and leptomeninges ${ }^{23}$.

The first place of cortical NSCs and NPCs is the anterior SVZ. Migrating new neurons from the anterior SVZ to the cerebral cortex have been observed in healthy animals ${ }^{8,10}$, although the number of newborn neurons is quite small in these studies. Interestingly, pathological treatments, such as ischemia, artificial neural degeneration, and aspiration lesion, seem to reroute new neurons from the anterior SVZ to damaged regions of the cerebral cortex $x^{15,17,18}$.

The second place is the white matter of the cerebral cortex. NSCs are isolated from the white matter of the adult human brains ${ }^{19}$. The isolated NSCs generate neurospheres, which give rise to neurons and glial cells both in vitro and after transplantation to the fetal rat brains. The brain samples are surgically taken from patients with epilepsy, arterial aneurysm, and traumatic injury, so that these NSCs might be induced by neural damages. In fact, the laserlesions activate endogenous NSCs and NPCs in the white matter of the cerebral cortex of adult rats ${ }^{20}$.
The third place is the gray matter of the cerebral cortex. In the gray matter of the cerebral cortex, there are NG2positive cells, which seem to generate cortical new neurons ${ }^{9}$. Newly-generated neurons are classified as GABAergic interneurons that express GABA-related molecules, such as GABA, GAD67, calretinin, and calbindin. In the SVZ and HDG, NG2-positive cells have been also reported to act as $\mathrm{NPCs}^{24-26}$. However, some studies have reported that NG2positive cells do not produce new neurons at all, by using genetically modified mice ${ }^{27-30}$. Further studies to examine whether these progenitors generate neurons, glial cells, or both cell types are needed.

The fourth place is the cortical layer 1 , the most superficial layer of the cerebral cortex. A few studies have reported that NSCs and NPCs are distributed in the cortical layer 1 . The focal laser-lesion of the rat visual cortex induces NSCs/NPCs in the layer $1^{20}$. Similar NSCs/ NPCs are induced in the layer 1 by spreading depression treatment ${ }^{21}$. The NSCs/NPCs are defined as the vimentinor nestin-positive cells. However, the two reports described above cannot provide direct evidence that new neurons are produced from the vimentin- or nestin-positive NSCs/ NPCs, because these data are based on immunohistological data. The direct labeling method of progenitor cells with GFP-expressing retrovirus vectors has identified NPCs in the layer 1 of the adult rodents ${ }^{16,31}$. NPCs in the layer 1 produce subclasses of GABAergic interneurons, and the layer 1 NPCs are designated as L1-INP cells (layer 1 inhibitory neuron progenitor cell).

The fifth place is the perivascular regions in the cerebral cortex. Recently, one study found that NSCs exist around perivascular regions in the cerebral cortex. The NPCs were located around blood vessels within poststroke areas and immunoreactive for the NSC marker nestin and some pericytic markers ${ }^{22}$.

The last place is the leptomeninges surrounding the cerebral cortex. The leptomeninges seem to contain some nestin-positive NSCs. These cells were produced by amygdala kindling, and further repeated stimulus of amygdala generated inhibitory and pyramidal neurons in the cerebral cortex ${ }^{23}$.

As the reason why NSCs and NPCs are located in various places, one possibility would be multistep cellular reactivity for depending on the strength and area sizes of the damages of the cerebral cortex. For example, in brains under healthy conditions, new neurons are generated at very low levels from NSCs and NPCs in gray matter9. After animals are subjected to a mild injury, such as kindling and ischemic insult resulting from a 10-min occlusion of both common carotid arteries, NSCs/NPCs in the cortical layer 1 and leptomeninges generate new neurons. More intense injuries, such as 
focal cerebral ischemia caused by 90 -min or permanent clamp (7-90 days until perfusion) $)^{17,32}$, aspiration- ${ }^{18}$ or laser-lesion ${ }^{20}$ of the cerebral cortex, and chromophoretargeted neuronal degeneration ${ }^{13,15}$, cause the generation of new neurons from the SVZ, perivascular regions, gray and white matter, and layer 1 . Thus, if NSCs and NPCs with different responsiveness for brain damage or stimulus exist in several places of the cerebral cortex, the cerebral cortex will be able to react to any damage or stimulus.

\section{Regulating Factors of Cortical NSCs and NPCs}

Recent studies have been clarifying that adult neurogenesis in the SVZ and HDG can be regulated by various factors; exercise, environmental enrichment, pregnancy, antidepressant treatments, and brain damages up-regulate neurogenesis, while stress and aging downregulate $\mathrm{it}^{33}$. Similar to adult neurogenesis in the SVZ and HDG, it is becoming clear that there are a few factors that can alter cortical neurogenesis. As described above, brain damages have the promoting effects of cortical adult neurogenesis. Besides brain damages, administration of antidepressant drugs and aging has been known as regulating factors of cortical adult neurogenesis.

The antidepressant fluoxetine (FLX) is one of the most widely used drugs for treating depression and anxiety disorders. Interestingly, chronic FLX treatment can change adult neurogenesis; upregulation of the HDG adult neurogenesis ${ }^{34}$ and down regulation of the SVZ adult neurogenesis ${ }^{35}$. Previous studies have found NSCs and NPCs in some subregions of the cerebral cortex, as described above. Of these regions, NPCs in the layer 1 at least have been shown to respond to chronic FLX treatment ${ }^{4}$. FLX treatment for 3 weeks increased the number of NPCs in the cortex in a dose-dependent manner and up-regulated production of inhibitory interneurons in all cortical layers in the prefrontal cortex of adult mice 4 . However, it is unknown whether the increase of cortical adult neurogenesis by FLX has an antidepressive effect, so future studies are needed to determine this.

Two reports demonstrated the presence of adult neurogenesis in the aged cerebral cortex. One study found that, during aging in mice, the densities of NPCs decreased in the layer 1 of the cerebral cortex ${ }^{36}$. The number of NPCs was kept by 12-month-old, dramatically decreased at 17-month-old, and maintained the same level until 24-month-old (Figure 1). NPCs in the aged cerebral cortex (24-month-old) have the capacity to produce new neurons by ischemia, the levels of which were higher than those of young controls (5 month-old). The other finding was derived from elderly humans ${ }^{22}$. NSCs were isolated from the brain samples obtained from the poststroke areas in two patients (79 and 81 years females). Interestingly, NSCs isolated from patients with stroke can differentiate not only to neurons, but also to osteoblasts, adipocytes, and chondrocytes, under osteoblastic, adipogenic, and chondrocytic differentiation conditions, respectively. The multipotent NSCs are likely derived from brain pericytes localized near blood vessels. Although cortical adult neurogenesis decreases with age, NSCs and NPCs are present in the aged cerebral cortex and the neurogenic capacity of the cerebral cortex, especially damage-dependent neurogenesis, might be maintained during aging.

Exercise, enrichment, stress, learning and memory, and the like are known as regulating factors influencing adult neurogenesis of the HDG and SVZ. It is necessary to investigate whether cortical adult neurogenesis changes due to these factors.

\section{Functions of Cortical Adult Neurogenesis}

For the last decade, studies of cortical adult neurogenesis have made steady progress, however, its functions remain to be largely unknown. One hint is that there are many reports that cortical adult neurogenesis is upregulated after brain damages, such as ischemia, epilepsy, neural degeneration, and lesion. These findings suggest that cortical adult neurogenesis has some functions against brain injury. Importantly, enhanced neurogenesis in the cerebral cortex may have neuroprotective effects ${ }^{4}$. When FLX increased the production of new inhibitory interneurons from NPCs in advance of forebrain ischemia, neuronal cell death around new neurons significantly decreased compared with the controls $^{4}$. FLX is a antidepressant drug and has been also reported to have neuroprotective effects on brain infarction in rodents and humans. The finding might suggest that new inhibitory interneurons suppress neuronal cell death in the infarction regions. Similar to the above finding, mice genetically deleted nestin-positive cells in the leptomeninges showed high susceptibility to electrical stimulation $^{23}$. Consequently, almost all of the mice without nestin-positive cells died or dropped out during kindling progression in 20 days later, suggesting that nestinpositive cells activated by amygdala kindling might be involved in neuroprotection against epilepsy. These results may suggest that brain tolerance to brain injury increases as new cells are produced in the cerebral cortex. Future studies may reveal the molecular and cellular mechanisms underlying neuroprotection by new cells, which may lead to a new treatment for brain injury.

\section{Cell Therapy in the Cerebral Cortex}

It was not believed enough, just ten years ago, that new neurons were produced in the cerebral cortex. Currently, increasing evidence supports adult neurogenesis in the cerebral cortex in humans as well as rodents. In the nervous system, researches on regenerative medicine are being actively conducted, by using iPS cells and ES cells. In addition to these stem cells, cell therapy using endogenous NSCs 
A

Primary motor cortex at 5 months of age

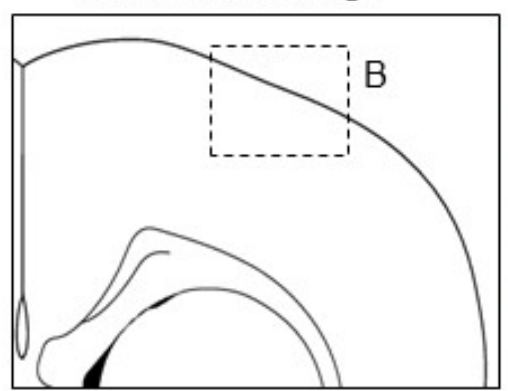

C
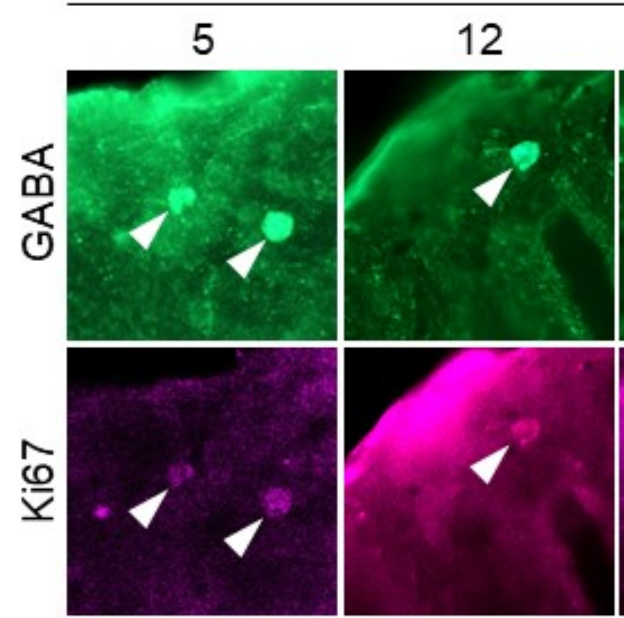

Month-old

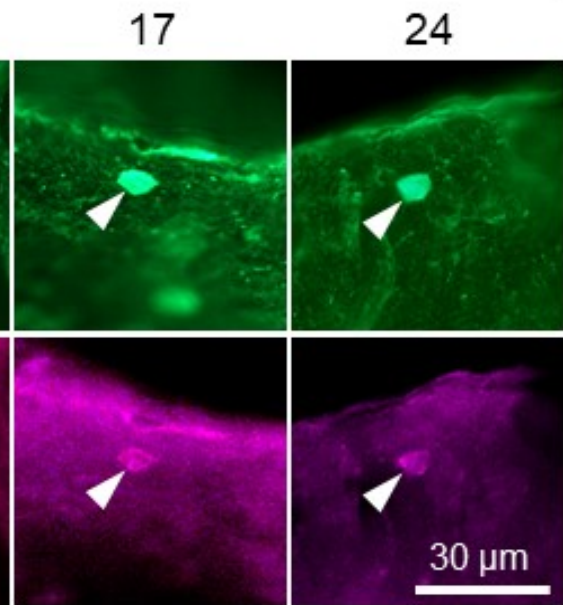

$\mathrm{D}$
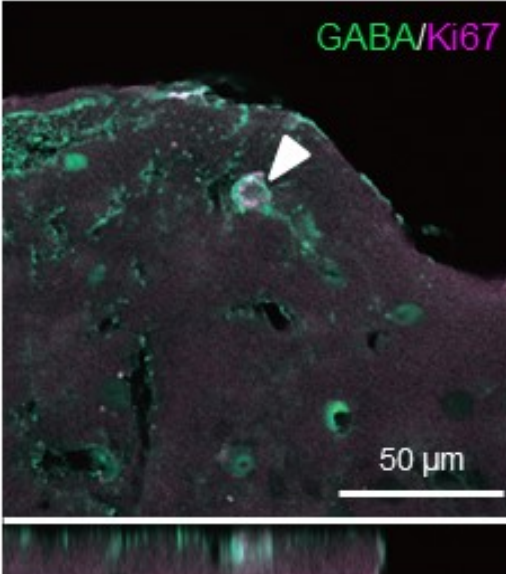

B

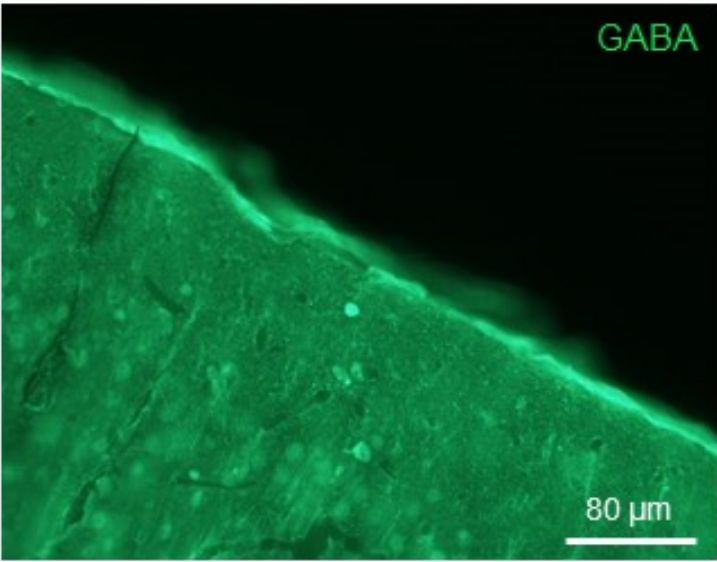

$80 \mu \mathrm{m}$ $\mu \mathrm{m}$

Figure 1: Changes of the numbers of L1-INP cells during aging in the cerebral cortex.

Schematic representation of the cerebral cortex at 5-month-old. A boxed area indicates the area of (B). The drawing was quoted from the mouse brain atlas ${ }^{37}$. (B) GABA-immunostained cells in the primary motor cortex at 5-month-old. (C) Representative images of L1INP cells during aging of the cerebral cortex. Arrowheads indicate L1-INP cells, which are labeled with anti-GABA (green) and anti-Ki67 (magenta). (D) A Z-plane of serial optical planes of L1-INP cell in the cerebral cortex of 5-month-old mice. L1-INP cells, which is marked by arrowheads, are stained with anti-GABA (green) and anti-Ki67 (red) antibodies. (E) Quantification of the numbers of L1-INP cells during aging in the cerebral cortex. The data are shown as means \pm S.E.M. ${ }^{* * * *}, \mathrm{P}<0.0001$. Values are analyzed by one-way ANOVA and Tukey's post hoc test, $\mathrm{n}=4$ each. 
and NPCs can be applied to the cerebral cortex. Although few functional studies have been carried out, activation of endogenous NSCs and NPCs in the cerebral cortex might reduce the loss of the cortical functions with aging. In particular, preventive effects can be expected in treatments using the endogenous NSCs and NPCs. In this case, how to activate endogenous NSCs and NPCs may be mentioned as an important technique. One hint is that L1-INP cells can be increased and differentiated with antidepressant treatments ${ }^{4}$. In other words, it is possible to manipulate the proliferation, suppression, and/or differentiation of endogenous NSCs and NPCs by administering substances, such as drugs and nutrients. Thus, we can say that research on adult neurogenesis of the cerebral cortex has changed from the study that clarifies its own existence to one that clarifies its functions. It is necessary to identify what factors have proliferative, differentiative, and inhibitory effects on endogenous NSCs and NPCs in the cerebral cortex at each life stage.

\section{Acknowledgments}

This work was supported by KAKENHI (17K07084) from the Japan Society for the Promotion of Science and Takeda Science Foundation.

\section{References}

1. Gross CG. Neurogenesis in the adult brain: death of a dogma. Nat Rev Neurosci. 2000; 1: 67-73.

2. Ming G, Song H. Adult Neurogenesis in the Mammalian Central Nervous System. Annu Rev Neurosci. 2005; 28: 223-250.

3. Ohira K. Injury-induced neurogenesis in the mammalian forebrain Cell Mol Life Sci. 2011; 68: 1645-1656.

4. Ohira K, Takeuchi R, Shoji H, et al. Fluoxetine-induced cortical adult neurogenesis. Neuropsychopharmacology. 2013; 38: 909-920.

5. Allen E. The cessation of mitosis in the central nervous system of the albino rat. J Comp Neurol. 1912; 22: 547-568.

6. Altman J. Autoradiographic investigation of cell proliferation in the brains of rats and cats. Anat Rec. 1963; 145: 573-591.

7. Kaplan MS. Neurogenesis in the 3-month-old rat visual cortex. J Comp Neurol. 1981; 195: 323-338.

8. Bernier PJ, Bedard A, Vinet J, et al. Newly generated neurons in the amygdala and adjoining cortex of adult primates. Proc Natl Acad Sci U S A. 2002; 99: 11464-11469.

9. Dayer AG, Cleaver KM, Abouantoun $\mathrm{T}$, et al. New GABAergic interneurons in the adult neocortex and striatum are generated from different precursors. J Cell Biol. 2005; 168: 415-427.

10. Gould E, Reeves AJ, Graziano MS, et al. Neurogenesis in the neocortex of adult primates. Science. 1999; 286: 548-552.

11. Gould E, Vail N, Wagers M, et al. Adult-generated hippocampal and neocortical neurons in macaques have a transient existence. Proc Natl Acad Sci U S A. 2001; 98: 10910-10917.

12. Koketsu D, Mikami A, Miyamoto Y, et al. Nonrenewal of neurons in the cerebral neocortex of adult macaque monkeys. J Neurosci. 2003; 23: 937-942.

13. Chen J, Magavi SSP, Macklis JD. Neurogenesis of corticospinal motor neurons extending spinal projections in adult mice. Proc Natl Acad Sci U S A. 2004; 101: 16357-16362.

14. Jiang W, Gu W, Brännström T, et al. Cortical neurogenesis in adult rats after transient middle cerebral artery occlusion. Stroke. 2001; 32: 1201-1207.

15. Magavi SS, Leavitt BR, Macklis JD. Induction of neurogenesis in the neocortex of adult mice. Nature. 2000; 405: 951-955.

16. Ohira K, Furuta T, Hioki H, et al. Ischemia-induced neurogenesis of neocortical layer 1 progenitor cells. Nat Neurosci. 2010; 13: 173-179.

17. Jin K, Sun Y, Xie L, et al. Directed migration of neuronal precursors into the ischemic cerebral cortex and striatum. Mol Cell Neurosci. 2003; 24: 171-189.

18. Sundholm-Peters NL, Yang HKC, Goings GE, et al. Subventricular zone neuroblasts emigrate toward cortical lesions. J Neuropathol Exp Neurol. 2005; 64: 1089-1100.

19. Nunes MC, Roy NS, Keyoung HM, et al. Identification and isolation of multipotential neural progenitor cells from the subcortical white matter of the adult human brain. Nat Med. 2003; 9: 439-447.

20. Sirko S, Neitz A, Mittmann T, et al. Focal laser-lesions activate an endogenous population of neural stem/progenitor cells in the adult visual cortex. Brain J Neurol. 2009; 132: 2252-2264.

21. Xue JH, Yanamoto $H$, Nakajo $Y$, et al. Induced spreading depression evokes cell division of astrocytes in the subpial zone, generating neural precursor-like cells and new immature neurons in the adult cerebral cortex. Stroke. 2009; 40: e606-613.

22. Tatebayashi K, Tanaka Y, Nakano-Doi A, et al. Identification of multipotent stem cells in human brain tissue following stroke. Stem Cells Dev. 2017; 26: 787-797.

23. Ninomiya S, Esumi S, Ohta $\mathrm{K}$, et al. Amygdala kindling induces nestin expression in the leptomeninges of the neocortex. Neurosci Res. 2013; 75: 121-129.

24. Aguirre A, Gallo V. Postnatal neurogenesis and gliogenesis in the olfactory bulb from NG2-expressing progenitors of the subventricular zone. J Neurosci. 2004; 24: 10530-10541.

25. Aguirre AA, Chittajallu R, Belachew S, et al. NG2-expressing cells in the subventricular zone are type C-like cells and contribute to interneuron generation in the postnatal hippocampus. J Cell Biol. 2004; 165: 575-589.

26. Belachew S, Chittajallu R, Aguirre AA, et al. Postnatal NG2 proteoglycanexpressing progenitor cells are intrinsically multipotent and generate functional neurons. J Cell Biol. 2003; 161: 169-186.

27. Dimou L, Simon C, Kirchhoff F, et al. Progeny of Olig2-expressing progenitors in the gray and white matter of the adult mouse cerebral cortex. J Neurosci. 2008; 28: 10434-10442.

28. Komitova M, Zhu X, Serwanski DR, et al. NG2 cells are distinct from neurogenic cells in the postnatal mouse subventricular zone. J Comp Neurol. 2009; 512: 702-716.

29. Nishiyama A, Komitova M, Suzuki R, et al. Polydendrocytes (NG2 cells): multifunctional cells with lineage plasticity. Nat Rev Neurosci. 2009; 10: 9-22.

30. Platel JC, Gordon V, Heintz T, et al. GFAP-GFP neural progenitors are antigenically homogeneous and anchored in their enclosed mosaic niche. Glia. 2009; 57: 66-78.

31. Ohira K, Kaneko T. Injection of virus vectors into the neocortical layer 1. Protoc Exch. 2010;10.1038/nprot.2010.21.

32. Leker RR, Soldner F, Velasco I, et al. Long-lasting regeneration after ischemia in the cerebral cortex. Stroke. 2007; 38: 153-161.

33. Abrous DN, Koehl M, Le Moal M. Adult neurogenesis: from precursors to network and physiology. Physiol Rev. 2005; 85: 523-569. 
34. Malberg JE, Eisch AJ, Nestler EJ, et al. Chronic antidepressant treatment increases neurogenesis in adult rat hippocampus. J Neurosci. 2000; 20: 9104-9110.

35. Ohira K, Miyakawa T. Chronic treatment with fluoxetine for more than 6 weeks decreases neurogenesis in the subventricular zone of adult mice. Mol Brain. 2011; 4: 10
36. Okada Y, Ohira K. Population dynamics of neural progenitor cells during aging in the cerebral cortex. Biochem Biophys Res Commun. 2017; 493: 182-187.

37. Paxinos G, Franklin KBJ. The mouse brain in stereotaxic coordinates: compact second edition. Academic Press. 2014. 